\title{
Metabolic Syndrome: Early Development and Aging
}

\section{Guarner Veronica* and Rubio-Ruiz Maria Esther}

Department Of Physiology, Instituto Nacional De Cardiologia “Ignacio Chavez”, México

\begin{abstract}
Adult metabolic and heart diseases have their origin early during intrauterine development and childhood. They can be traced to the inherited genome but patterns of cellular gene expression appropriate to the environment during development (epigenetics) also play an important role. This is particularly important during critical periods of time where structural and functional changes known as critical windows. There is a critical window of the pancreas when, if changes in diet occur, the risk of metabolic diseases during adulthood increases.

Aging is defined as a series of morphological and functional changes which take place over time. The term also refers to the deterioration of the biological functions after an organism has attained its maximum reproductive potential. The aging process is altered or accelerated when cardio-metabolic diseases are present but also aging makes organisms more susceptible to such ills. The same genes and proteins that might undergo epigenetic changes that predispose to metabolic diseases in the adult, also participate in the acceleration of aging with metabolic syndrome. Aging predisposes to metabolic syndrome, type-2 diabetes or insulin resistance; however, other predisposing conditions such as obesity, inflammation, changes in the activity of the hypothalamus-hypophysis suprarenal axis and in the activity of the autonomous nervous system, hypertension, also contribute to increase its prevalence.
\end{abstract}

Keywords: Metabolic syndrome; Early development; Epigenetic changes; Critical windows; Aging; Insulin resistance; Hypertension

\section{Early Development}

It is nowadays recognized that adult metabolic and heart diseases, begin early during intrauterine development and childhood. Many of them can be traced to the inherited genome but patterns of cellular gene expression appropriate to the environment during development (epigenetics) also play an important role [1]. Such nongenomic tuning of phenotype through developmental plasticity has adaptive value because it attempts to match an individual's responses to the environment predicted to be experienced. When the responses are mismatched, disease risk increases. Environmental influence on the genome is particularly important during critical periods of time where structural and functional changes during normal organ development known as critical windows [2]. Therefore it is important to consider the early development of an individual for a better understanding of cardio-metabolic diseases.

\section{Epigenetics}

The term epigenetics refers to processes which establish and maintain distinct patterns of cellular gene expression appropriate to the environment during development and that are mitotically heritable without changing the underlying gene sequence [1]. The epigenetic processes involving chromatin remodeling have received much attention in the last decade and play important roles in contemporary research in animal physiology. They consist of: A) DNA methylation which comprises the addition of a methyl group to nucleotides, which typically silences gene expression. Mammalian gene promoters contain cytosine-guanosine dinucleotide $(\mathrm{CpG})$ sites which play a pivotal role in the control of gene expression. Methylation of the cytosine residues regulates transcription directly by inhibiting the binding of specific transcription factors, and indirectly by recruiting methyl-CpG-binding proteins and their associated repressive chromatin-remodeling activities. These epigenetic alterations are responsible for modulation of developmentally-regulated and tissue-specific gene expression $[3,4]$. B) Histone modification which consists of the acetylation mediated by histone deacetylases (HDAC) and/or methylation of chromosome packaging proteins, C) Noncoding RNA (ncRNAs) activity, involving small RNAs, microRNAs and large RNAs, which has also been shown to play an important role in modulating protein activity via regulation of translation, transcription or protein structure. There is increasing evidence for regulatory roles of ncRNAs during development and in response to stress and environmental stimuli. MicroRNAs play a role in many diseases, including diabetes $[5,6]$. D) Parental effects generally can be defined as the change in offspring phenotype due solely to maternal and/or paternal influence without a concomitant change in offspring DNA sequence [7].

These changes result in life-long alterations in gene expression. Such nongenomic tuning of phenotype through developmental plasticity has adaptive value because it attempts to match an individual's responses to the environment predicted to be experienced. When a mismatch arises the risk of diseases increase as for example when either there are inaccurate nutritional cues from the mother or placenta before birth, or there is a rapid environmental change through improved socioeconomic conditions, these changes would contribute to the increasing prevalence of type-2 diabetes, obesity, and cardiovascular disease [1].

During early embryogenesis, the mammalian genome is 'wiped clean' of most epigenetic modifications, which are progressively reestablished during embryonic development. Thus, the epigenome of each mature cellular lineage carries the record of its developmental history. The subsequent trajectory and pattern of development are also

*Corresponding author: Verónica Guarner Ph.D, Departamento de Fisiología Instituto Nacional de Cardiología "Ignacio Chávez", Juan Badiano 1, Tlalpan, México, D.F 14080, México, Tel: 52-5-573-29-11; Fax: 52-5-573-09-94; E-mail gualanv@yahoo.com

Received October 11, 2011; Accepted November 20, 2011; Published November 24, 2011

Citation: Veronica G, Esther RRM (2011) Metabolic Syndrome: Early Development and Aging. J Diabetes Metab S2:002. doi:10.4172/2155-6156.S2-002

Copyright: (C) 2011 Veronica G, et al. This is an open-access article distributed under the terms of the Creative Commons Attribution License, which permits unrestricted use, distribution, and reproduction in any medium, provided the original author and source are credited. 
responsive to environmental influences, and such plasticity is likely to have an epigenetic basis. Epigenetic marks may be transmitted across generations, either directly by persisting through meiosis or indirectly through replication in the next generation of the conditions in which the epigenetic change occurred [8].

A definition of "epigenetic epidemiology" has been proposed and it is important to delineate how this emerging field can provide a basis from which to explore the role of epigenetic mechanisms in "developmental origins of health and disease" (DOHaD) [9].

\section{Epigenetics of metabolic syndrome}

There is evidence for the induction of different phenotypes by variations in the early life environment, including nutrition, which in man is associated with a graded risk of metabolic disease [10-13]. That there is a heritable or familial component of susceptibility to chronic non-communicable diseases such as type- 2 diabetes, obesity and cardiovascular disease, is well established, but there is increasing evidence that some elements of such heritability are transmitted nongenomically and that the processes whereby environmental influences act during early development to shape disease risk in later life can have effects beyond a single generation [14].

Insulin is a master regulator of metabolic homeostasis and it is secreted from pancreatic beta cells in response to nutrient stimulation. Insulin gene expression begins early in the embryonic development of the pancreas and remains tightly-regulated at multiple levels throughout adult life. Previous studies have established the regulation of insulin activity at the transcriptional, post-transcriptional, translational, secretory, and even post-receptor levels. In mice and humans expression and secretion of insulin are epigenetically controlled by methylation of specific $\mathrm{CpG}$ sites in the promotor region of the gene. Additionally, miRNAs play an important role in the production and secretion of this hormone and levels of several miRNA in beta cells are regulated by nutrients such as glucose $[4,6]$.

\section{Maternal lifestyle and epigenetics}

Maternal obesity might adversely affect fetal development, producing lasting effects on offspring, including predisposition to obesity and diabetes. Obesity and insulin resistance are closely linked. A growing body of evidence demonstrates that obesity and insulin resistance have a fetal origin in many patients.

Low-grade inflammation accompanies obesity. Maternal obesity induces fetal inflammation, which changes fetal skeletal muscle development by promoting adipogenesis. Obesity activates the JNK signaling pathway. JNK signaling activates JUN, which induces expression of inflammatory-related genes. Recent evidence points to the association between inflammation and epigenetic modification of key genes regulating myogenesis and adipogenesis, providing an additional mechanism for inflammation and altered fetal skeletal muscle development [15].

Stressful conditions and inflammation in the mother increase NF- $\kappa B$ activity and elevate expression of pro-inflammatory cytokines which increase activation HDAC. Although HDAC inhibitors increase acetylation of histones, a condition associated with increased transcriptional accessibility, multiple reports have shown that HDAC inhibitors possess suppressive effects on immune response gene induction. HDAC inhibitors decreased TNF- $\alpha$-induced monocyte adhesion in vitro and in vivo via suppression of the VCAM-1 gene $[16,17]$. Therefore HDAC inhibitors have been increasingly identified as possible therapeutic approaches toward many inflammatory conditions, including cardiovascular diseases [18]. Therefore stressful conditions in the mothers might induce epigenetic changes in the offspring leading to increased risk of metabolic diseased during adulthood.

\section{Low birth weight and metabolic syndrome}

Clinical observations indicate there are long-term health consequences for persons of low birth weight. Neel [19] proposed the first theory to explain this relationship based on the existence of a diabetic genotype known as the "economic or thrifty genotype". It was based on epidemiologic studies in west North America where a high incidence of type- 2 diabetes is observed and it proposed that "diabetic or economic genes" were selected during evolution from stages in which food was scarce. These genes favored that, after energy requirement fulfillment, the surplus substrates would be accumulated as fat to provide energy during long fasting periods until new food would be available. This condition that was beneficial during fasting periods is nowadays a predisposing condition to the development of insulin resistance, type- 2 diabetes and obesity.

Some years later a complementary theory was proposed. Hales \& Barker [20] explained the association of nutrient deficiency and diabetes during adult life, based on the "theory of programming". They proposed that high insulin-mediated glucose utilization leads to the organic overwork of the pancreas in undernourished individuals during early developmental stages. Substrates in these individuals are destined to be used by the brain, leaving unprotected organs such as muscle, liver and pancreas thus leading to altered metabolism during postnatal life. The individual is programmed to survive in precarious conditions and when these conditions change the adaptations predispose to metabolic diseased. This theory is in accordance to epigenetic explanations $[21,22]$.

Examples of genes that are "epigenetically susceptible" being affected by perinatal insults have been reported such as the dualspecificity phosphatase 5 (DUSP5) and insulin-like growth factor (IGF-1). DUSP5 is a nuclear Erk-specific phosphatase which negatively regulates members of the mitogen-activated protein (MAP) kinase superfamily (ERK-1 and ERK-2) and also has as a target the insulin receptor substrate 1, a major component of the insulin signaling pathway. Alterations in histone acetylation and methylation of specific CpG sites of the DUSP5 gene are related to presence of insulin resistance in the adult $[23,24]$.

IGF-1 is of vital importance for growth, normal carbohydrate metabolism in both mice and humans, and mediates many of the anabolic and mitogenic actions of GH in postnatal life. Low IGF-1 levels also contribute to adult insulin resistance [24,25].

\section{Epidemiological Studies Relating Low Birth Weight to Disease Predisposition}

Forsdahl [26] in the United Kingdom was the first to report a correlation between a nutritional deficit during childhood and an increased risk to develop diseases during adulthood. Wadsworth [27] reported that blood pressure was inversely correlated with birth weight in men and women born in 1946. In 1987, in Hertfordshire, one of the first retrospective studies was done with subjects born between 1911 y 1930, showing low weight at birth or during their first year of life. The results were published by Barker [28] and he showed a high incidence of hypertensive persons and people dying from cardio-vascular diseases. Hales [20] published that the same subjects also showed 
altered glucose intolerance tests or type-2 diabetes. The rate of these conditions decreased in people who had higher body weight at birth. In India Yajnik $[29,30]$ proposed that these diseases are also increased if there is an um-balanced diet during childhood or a change in lifestyle.

The Pima Indians are an isolated community which lives in Arizona and are genetically related to Mexican communities living in the Sierra Madre in Mexico. This community has a high incidence of insulin resistance and type-2 diabetes [31]. In contrast to inhabitants of the Sierra Madre the community in the United Status shows obesity due to elevated calorie ingestion and low energy expenditure [31,32]. The early influence of diet during early childhood and genetic factors also play an important role in the development of insulin resistance and type-2 diabetes [33]. Numerous recent epidemiological studies associate early nutrition and low birth weight to an increased risk to develop obesity, diabetes and hypertension during adulthood [34-40].

\section{Critical windows}

Multiple studies demonstrate that poor in utero growth predicts adult morbidities, such as obesity and insulin resistance. There is growing evidence, primarily from animal studies, that supports the idea that processes put in motion during development that contribute to $\mathrm{DOHaD}$ do not necessarily reflect compromised growth or altered birth weight. Throughout the body of work investigating the $\mathrm{DOHaD}$ hypothesis, several themes have emerged, particularly the presence of critical periods of vulnerability.

The process of DNA methylation takes place in utero and in early postnatal development and is therefore a good candidate for disturbance by environmental interference, and thus provides a potential mechanism for fetal programming. Whereas methylation patterns are generally considered to be established in early postnatal life and persist thereafter, there is evidence from human monozygotic twin studies that methylation patterns can change with aging.

A critical window is a period of important structural and functional changes during normal organ development when exposure to certain environmental changes may originate life-time consequences [2].

Lucas [41] proposed the "programming theory" which describes how a dietary deficiency or imbalance might be kept in the memory of cells or individuals for the rest of a lifetime or even be transmitted to the next generation. This programming consists of a saving fenotype leading to more fat accumulation and a propensity to develop obesity and metabolic diseases and was afterwards named "metabolic or nutritional programming" [42]. In contrast to Lucas, Waterland [43] suggested that more than a "metabolic programming" it is a "metabolic print" leading to a "thrifty or economic fenotype".

Since the earliest stages of evolution, organisms have faced the challenge of sensing and adapting to environmental changes for their survival under compromising conditions such as caloric restriction (CR) or stress. Implicit in these responses are mechanisms developed during evolution that include the targeting of chromatin to allow or prevent expression of fundamental genes and to protect genome integrity. CR is tightly related with the structure, stability and function of the chromatin of specific tissues and that it regulates several processes such as aging [44]. One of the factors by which CR induces changes in the chromatin is the $\mathrm{NAD}^{+}$-dependent deacetylases known as sirtuins (SIRT1-7 in mammals). The sirtuins are a family of proteins localized in various subcellular compartments that have been implicated in longevity, metabolic processes and stress resistance. Sirtuins are a highly conserved group of $\mathrm{NAD}^{+}$-dependent deacetylases and their dependence on $\mathrm{NAD}^{+}$suggests that they might be possible mediators of dietary restriction. NADH has been suggested to be more important to nutrient sensing than ATP.

In mammals, SIRT1 deacetylates many key transcription factors and cofactors, such as the tumor suppressor p53, forkhead box class $\mathrm{O}$ (FOXO) proteins, peroxisome proliferator- activated receptor- $\gamma$ coactivator-1a (PGC-1 $\alpha$ ), and nuclear factor- $\kappa \mathrm{B}$. These specific actions may affect cellular pathways involved in glucose homeostasis. The effects of SIRT appear to be beneficial, as they trigger metabolic changes similar to those observed in CR. Indeed, CR increases the levels of SIRT1 in liver and muscle, which are key insulin-sensitive organs. In light of these observations, SIRTs have been proposed as a possible target for the treatment of metabolic syndrome (MS) [45].

\section{The critical window of the pancreas}

A postnatal critical window for the rat pancreas has been demonstrated [11]. The nutritional status during the breastfeeding period which corresponds to the pancreatic critical window, determines the development of glucose intolerance in adulthood and in turn predispose to development of type-2 diabetes [12]. In humans, critical windows also occur but it is difficult to identify them because they begin at earlier stages and continue for longer after birth [46].

The first month of life in the rat determines glucose homeostasis. During this period, rat pups are mother-fed with milk until day 21 when weaning occurs. Blood glucose and insulin levels are elevated in the rat during the suckling period and decrease after weaning [11]. Circulating levels of cortisol could cause the high glucose levels since this hormone induces a reversible loss of glucose sensitivity $[11,47]$. The insulin tolerance test in 20 day old rats is flat when compared to the adult [11]. Glucagon decreases throughout the first month and from day 6 to day 12 glucagon levels are higher than those of insulin. A decrease in the insulin/glucagon ratio induces glycogenolysis, gluconeogenesis and ketone body synthesis $[48,49]$. Therefore this neonatal period is characterized by physiologic hyperglycemia and hyperinsulinemia and by insulin resistance.

If the diet is changed during the suckling period in the rat, hyperinsulinemia and hyperglucagonemia are observed [50,51], beta cell secretory function is altered [12,52] and morphological changes within the pancreas are observed [53]. These changes program the pancreatic islet function in adult rats, resulting in the maintenance of hyperinsulinemia in the post-weaning period and eventually leading to the development of obesity in adult life [51,52]. The nutritional status during the breastfeeding period in humans also determines the development of glucose intolerance in adulthood and in turn predispose to development of type-2 diabetes [13]. Furthermore, low birth weight in humans is strongly predictive not only of obesity and diabetes but of hypertension, cardiovascular diseases and insulin resistance [54,55].

An epigenetically regulated gene involved in linking maternal diet to development of disease during adult life of the offspring is the hepatocyte nuclear factor $4-\alpha(\mathrm{HNF}-4 \alpha)$. HNF-4a is a transcription factor from the nuclear hormone receptor superfamily, which plays a pivotal role in development of the fetal endocrine pancreas, adult pancreatic function, and, consequently, glucose tolerance. HNF- $4 a$ has been linked to the development of type 2-diabetes [56].

Hyperglicemia and hyperinsulinemia modify vascular responses [57]. MS rats which have hyperglicemia and hyperinsulinemia, hypertriglyceridemia and central obesity have increased vascular contraction to $\mathrm{KCl}$ and norepinephrine and decreased vasorelaxation 
when compared to control responses [58]. Insulin resistance is strongly associated to acute coronary syndrome in young people and limits endothelium dependent coronary vasodilation $[59,60]$. Hyperinsulinemia and insulin resistance are also present in rats which develop MS [61]. Changes in aortic vaso-reactivity during the postnatal pancreatic critical window, where insulin and glucose, are elevated have been studied and compared to those in control and MS rats [62].

\section{Is there a critical window for hypertension?}

It is nowadays recognized that adult heart diseases, coronary artery disease and essential hypertension begin in childhood, after studies of autopsies in which cardiovascular and renal modifications can be observed since the first decades of life. Therefore, risk factors can be identified from early development on and preventive measures can be taken from very early [63].

There is a tight correlation between adult blood pressure measured in children and their values when adulthood is reached [34]. Age, height, body mass, initial blood pressure levels and a family history of hypertension determine changes in blood pressure in children. Diet and levels of electrolytes also play a significant role in regulation of blood pressure in childhood. Cardiac output, left ventricular mass, renal blood flow and autonomic nervous system activity in children and young people correlate to increased blood pressure and future hypertension [64].

During the fetal stage, one of the mechanisms employed by the body to compensate a nutritional deficit is to protect vital organs shifting blood flow to favor important organs and diminishing blood flow to other regions [39]. The rennin-angiotensin system (RAS) is altered in low birth weight subjects [65]. Vessel structure is also modified having a reduced elasticity $[36,66]$. Cardiac myocytes which are completely differentiated before birth are also altered being bigger and less numerous [39].

Vascular dysfunction is present in many types of hypertension and in MS caused mainly by a decreased production or biodisponibility of nitric oxide. In mammals, the production of nitric oxide is catalyzed by 3 isoforms of nitric oxide synthase (NOS) encoded by separate genes on 3 different chromosomes: neuronal NOS (NOS1), inducible NOS (iNOS) (NOS2), and endothelial NOS (eNOS) (NOS3). These NOS isoforms differ in their regulation and cell-specific distribution. The latter isoform, eNOS, is constitutively expressed and responsible for the majority of nitric oxide produced by the vascular endothelium. Both eNOS and iNOS are examples of epigenetically regulated genes in which DNA methylation and histone posttranslational modifications determine the expression in cells at specific conditions. Aditionally, the expression of these enzymes is regulated by transcriptional, posttranscriptional and postraductional mechanisms [17].

Histone deacetylases, particularly HDAC3 and HDAC7, are necessary for vascular normal development and they regulate genetic expression in diseases where transcription factor NF- $\kappa \mathrm{B}$ is implicated [17].

The RAS also participates in many types of hypertension. Environmental and maternal factors regulate the expression of some of the components of this system through epigenetic modifications and therefore favor the development of hypertension [67].

A high-salt diet limited to the prenatal period is not sufficient to program hypertension in adult offspring. However, this narrower critical period is sufficient to imprint a lasting hyper-responsiveness to stress, in adult female offspring. These data indicate that excessive maternal salt intake during pregnancy can adversely affect the cardiovascular health of adult offspring [68].

\section{Aging}

Aging is defined as a series of morphological and functional changes which take place over time. The term also refers to the deterioration of the biological functions after an organism has attained its maximum reproductive potential. We do not know yet whether organisms start to grow old from the moment of conception or from maturity, or if aging constitutes a process of evolution or involution. Stress response genes and nutrient sensors regulate energy directed to cell protection and maintenance; when food is plentiful and stress levels are low, genes support growth and reproduction, in contrast harsh conditions favor a shift in gene activity towards cell protection and maintenance extending lifespan. Important genes in extending lifespan include kinase mammalian target of rapamycin (mTOR), AMP-activated protein kinase (AMPK), sirtuins and insulin/IGF-1 signaling. These genes integrate longevity pathways and metabolic signals in a complex interplay in which lifespan appears to be strictly dependent on substrate and energy bioavailability [69].

Both genome-wide and specific methylation profile/patterns change with age, and this may be genetically controlled. A generalized decrease in DNA methylation with age has been reported in mice and in cell lines, although this decrease may be tissue and/or gene specific. Decreased methylation may be accompanied by reactivation of previously silenced genes [70,71].

SIRT6 is a major neural histone deacetylase with important potential implications for common human diseases. Remarkably, SIRT6 deficiency causes dramatically increased acetylation of histones $\mathrm{H} 3 \mathrm{~K} 9$ and H3K56, despite the presence of several other histone deacetylases in the brain, including SIRT1 and SIRT2.

Schewer [72] link neural SIRT6 to GH/IGF1 signaling, a pathway conserved across phyla that affects lifespan, metabolism, cancer, and neurodegeneration and propose that SIRT6 functions as a central regulator of somatic growth and plays an important role in preventing obesity by modulating neural chromatin structure and gene activity.

IGF-1 mediated signalling is determining for longevity. Abnormalities in the insulin signaling pathway generate age-related diseases and increased mortality, whereas the GH/IGF-1 axis could potentially modulate longevity in many species. Moreover in humans, an age-related decline in IGF-1 levels occurs, and at old age, low IGF-1 levels are associated with frailty, poor nutrition and cognitive decline and an increased risk of death [73,74].

The aging process is altered or accelerated when metabolic and cardiovascular diseases are present and the risk of diseases increases with age. Many predisposing conditions which increase in prevalence during aging, such as obesity, insulin resistance, inflammation, changes in the activity of the hypothalamus-hypophysis suprarenal axis, stress and hypertension also contribute to increase prevalence of MS. Aging and the development of insulin resistance seem to be accelerated in the MS [75-77].

\section{Aging and metabolic syndrome}

The mechanisms that account for precocious aging in MS are incompletely known, but it is becoming clear that longevity genes might be involved. Experiments with overactivation or disruption of key lifespan determinant pathways, such as silent information regulator SIRT1, p66Shc, and mTOR, lead to development of features of the MS in 
mice. Additional pathways are involved in linking nutrient availability and longevity, certainly including insulin and IGF-1 signaling, as well as FOXO transcription factors [78]. The same genes and proteins participating in the accelerated aging in MS are the possible blanks undergoing epigenetic changes during early development.

Although there is considerable disagreement on the underlying MS pathophysiology, clinical and experimental data support a link between SIRT1 and MS. Interestingly, the only clinical variable related to SIRT1 beyond MS components was age, which is physiologically related to SIRT1 function as a life-span determinant gene [45].

\section{Aging and obesity}

Obesity and aging are two overlapping and mounting public health problems. The prevalence of obesity has been related to the increasing prevalence of MS, which is growing progressively even among older age groups. Indeed, Insulin resistance observed with aging may be more related to adiposity than aging per se [79]. Obesity is strongly correlated with insulin resistance, although not all obese patients have insulin resistance, nor does insulin resistance always result in obesity [80]. Obesity is linked to an increased production of inflammatory adipokines that may alter insulin sensitivity and muscle mass [81].

Central, or visceral, adiposity has been particularly associated with insulin resistance and MS, but the mechanisms responsible for this association are unclear [82]. Obesity leads to insulin resistance through the increased liberation of free fatty acids. Fatty acids interfere with glucose transporter translocation through alterations in protein kinase C $\lambda$ activity. They also down-regulate insulin receptor substrate 2 (IRS2) and increase the expression of the lipogenic transcription factor SREBP-1c [82].

Insufficient intracellular fat oxidation is an important contributor to aging-related insulin resistance, while the precise underlying mechanism is unclear. AMPK is an important regulator of intracellular fat oxidation and plays a key role in high-glucose and high-fat induced glucose intolerance [83].

It is well known that $\mathrm{CR}$, in many ways opposite to obesity, increases lifespan. Pathways activated by CR include insulin/IGF1 pathway affecting the downstream phosphatidylinostiol 3-kinase/ AKT/PDK kinase cascade, mTOR, AMPK and sirtuins [69]. When obesity is present, these pathways are regulated in the opposite direction accelerating aging.

\section{Aging, inflammation and metabolic syndrome}

Inflammation is a key factor in the progressive loss of lean tissue and impaired immune function observed in aging. Low-grade inflammation in adipose tissue contributes to insulin resistance and type-2 diabetes [84]. This conclusion is predominantly drawn from studies demonstrating associations between elevated (but 'normal range') levels of circulating acute phase inflammatory markers, typified by C-reactive protein (CRP), interleukin-6 and indices of insulin resistance and the development of type- 2 diabetes. There is a debate as to whether these associations are independent of body fatness or, rather, an epiphenomenon of central obesity, an important source of inflammatory cytokines [85].

In addition to producing more inflammatory cytokines, adipocytes from old mice induce a higher inflammatory response in other cells. Sphingolipid ceramide is higher in old than in young adipocytes. Reducing ceramide levels or inhibiting NF- $\kappa \mathrm{B}$ activation decreases cytokine production, whereas the addition of ceramide increases cytokine production in young adipocytes to a comparable level to that seen in old adipocytes, suggesting that ceramide-induced activation of NF- $\kappa B$ plays a key role in inflammation [84].

There are predisposing factors for the chronic inflammation that occurs during aging. These include increased oxidative stress, a decrease in ovarian function, a decrease in stress-induced glucocorticoid sensitivity of pro-inflammatory cytokine production in men, and an increased incidence of asymptomatic bacteriuria [86].

An elderly immune system becomes more and more predisposed to chronic inflammatory reactions and is less able to respond to acute and massive challenges by new antigens. A young immune system has to cope quickly and efficiently with acute immunological challenges to assure survival and the reaching of reproductive age. Such reaction capability gradually burns out because of lifelong antigenic attrition. Moreover, lifelong antigenic challenges and the increasing antigenic burden determine a condition of chronic inflammation, with increased lymphocyte activation and proinflammatory cytokines [86].

Polymorphisms in the promoter regions of pro- and antiinflammatory cytokine genes influence the level of cytokine production and the aging process. Nutrients with anti-inflammatory properties, such as vitamin $\mathrm{E}$ and $\mathrm{n}-3$ polyunsaturated fatty acid, may reduce the level of chronic inflammation and thereby ameliorate tissue and functional loss during aging. New evidence suggests that, for the latter nutrient, gene-nutrient interactions occur that alter the effectiveness of dietary therapy [87].

\section{Aging, central nervous and stress responses and their influence on metabolic syndrome}

During aging there is a decreased response to stress-induced glucocoticoid sensitivity. Aging has been associated with immunological changes that resemble those observed following chronic stress or glucocorticoid (GC) treatment. Immunosenescence could be associated with changes of peripheral GC levels [88].

Leptin, in addition to regulating appetite and energy expenditure, also regulates corticotropin function [89]. Weight gain increases feedback to the brain (via hyperleptinemia), which in turn results in hypothalamus-pituitary-adrenal axis (HPA-axis) and sympathetic nervous system overdrive, impaired insulin secretion and insulin resistance [90]. HPA-axis overdrive would account for metabolic abnormalities such as central adiposity, hyperglycemia, dyslipidemia, and hypertension, which are well known clinical aspects of the MS [90].

The HPA axis could be stimulated by central factors followed by discrete, periodical elevations of cortisol secretion during normal conditions. A prolonged period of HPA axis stimulation is followed by a continuous degradation of the regulatory mechanisms. The final stage is sustained cortisol secretion with low morning values [91,92].

Stress responsiveness is decreased during aging. The unavoidable chronic overexposure to stressors determines a highly pathogenic sustained activation of the stress-response system, leading to a progressively reduced capacity to recover from stress-induced modifications [86]. Glucocorticoids, the adrenal steroid hormones secreted during stress, may account for this relationship [93].

Stress is a potent modulator of immune function, which in youth can be compensated for by the presence of an optimal immune response. In the elderly the immune response is blunted as a result of the decline in several components of the immune system (immune senescence) and a shifting to a chronic pro-inflammatory status 
(the so-called 'inflamm-aging' effect) [94]. Aging is accompanied by a greater increase in sympathetic traffic in women than in men, and inflammation (measured via CRP) seems to be more strongly related to the MS in women than in men [95].

Sympathetic nerve fibers innervate white adipose tissue and stimulate lipolysis, leading to the release of glycerol and free fatty acids. There is also a functional parasympathetic innervation and a distinct somatotropy within the parasympathetic nuclei: separate sets of autonomic neurons in the brain stem innervate either the visceral or the subcutaneous fat deposit. Parasympathectomy induces insulin resistance associated with glucose and fatty acid uptake in the fat depot and has selective effects on local hormone synthesis $[96,97]$.

The autonomic nervous system modulates glucose and fat metabolism through both direct neural effects and hormonal effects. Locally released norepinephrine from sympathetic nerves is likely to increase glucose uptake in skeletal muscle and adipose tissues, independent of insulin, but norepinephrine does not contribute as much as epinephrine to hepatic glucose production. Alterations of central neurotransmission and environmental factors can change the relative contribution of sympathetic outflow to the pancreas, liver, adrenal medulla and adipose tissues, leading to the modulation of glucose and fat metabolism. Recent studies have proposed that leptin, an adipocyte hormone, induces the central nervous system to increase sympathetic outflow independently of feeding [82].

One of the genes involved in aging is mTOR. Its inhibition increases resistance to environmental stress, consistent with a physiological shift towards tissue maintenance and an increase in lifespan [69]. The decreased stress response during aging might be caused by mTOR's diminished expression.

Mammals have developed an endogenous circadian clock located in the suprachiasmatic nuclei of the anterior hypothalamus that responds to the environmental light-dark cycle. The circadian clock influences nearly all aspects of physiology and behavior, including sleep-wake cycles, cardiovascular activity, endocrine system, body temperature, renal activity, physiology of the gastrointestinal tract, and hepatic metabolism. Moreover, disruption of circadian rhythms leads to metabolic disorders like manifestations of the MS [98]. Circadian rhythms are altered during aging [99].

The clock machinery comprises complex transcriptionaltranslational feedback loops that, through the action of specific transcription factors, modulate the expression of as many as $10 \%$ of cellular transcripts. This marked change in gene expression necessarily implicates a global regulation of chromatin remodeling. Indeed, various descriptive studies have indicated that histone modifications (like acetylation) occur at promoters of clock-controlled genes (CCGs) in a circadian manner. Moreover SIRT1 is a central physiological and molecular link that connects chromatin remodeling to circadian and metabolic processes. SIRT1 appears to modulate circadian gene expression by repressing transcription through its HDAC activity in a time dependent manner, presumably by enhancing localized chromatin condensation [100].

\section{Aging and hypertension}

Diminished insulin sensitivity is a characteristic feature of various pathological conditions such as the MS, type-2 diabetes and hypertension [101]. There are twice as many hypertensive patients among diabetics than there are among non-diabetics [102].
Patients with essential hypertension are more prone than normotensive subjects to develop diabetes, and this propensity may reflect decreased ability of insulin to promote relaxation and glucose transport in vascular and skeletal muscle tissue $[101,103]$. Although the etiology of skeletal muscle insulin resistance is multifactorial, there is accumulating evidence that one contributor is overactivity of the RAS $[101,103]$

Numerous studies focus on comparing the differences in development and aging patterns between hypertensive and normal rats. The contractile responses of vascular smooth muscle to norepinephrine, $\mathrm{KCl}, 5$-hydroxytryptamine, electro-stimulation and calcium-free physiological solution are increased during development and maturity in normal rats. Endothelium-dependent relaxation mediated by acetylcholine as well as isoproterenol, which is mediated by the $\beta$-receptor, falls during rat maturation [104].

In an experimental hypertension model in rats, based on a fructose-rich diet, endothelin expression is increased in blood vessels, while nitric oxide levels are not modified [105]. In another rat model of hypertension obtained by administering $30 \%$ sucrose in the drinking water for 20 weeks, an increased insulin-dependent endothelin response of the vessels has been found [57]. The sucrose-fed rat becomes hypertensive, hypertriglyceridemic, hyperinsulinemic and also has insulin resistance; it develops MS [61]. Some characteristics of the aging process in this model have been reported. Sucrose-fed animals showed increased central adiposity, insulin was increased at 6 months in MS rats and this increase accompanied the maximal blood pressure and contractility. Values for all of these variables decrease after this age. Triglycerides were increased at all ages in the experimental group. Glucose was increased in both MS and control rats at 18 months and is therefore a consequence of the aging process [106]. In control rats, systolic blood pressure did not change at the ages studied. As expected, blood pressure in MS rats was higher than in control animals, showing a maximum value at 6 months of age; this value then diminished considerably and in very old rats (18 months), it was even lower than in control rats of the same age. Therefore rats clearly showed premature aging due to the MS [106].

\section{Future Perspectives and Conclusion}

The variability of the environment determines acquired characteristics through natural selection, epigenetic expression and current adaptive strategies. These lead to activity in metabolic pathways which influence the rate of aging. Diseases such as metabolic syndrome also modulate the activity in these pathways and therefore modulate longevity (Figure 1).

The finding of epigenetic factors involved in MS pathogenesis has favored the development of new therapeutic measures. AMPK has been a target for treatment of type-2 diabetes and MS using drugs that stimulate its activity such as metformin or tiazolinedinediones. AMPK regulates transport and oxidation of fatty acids, mithochondrial biogenesis in skeletal muscle and insulin synthesis and secretion by phosphorylating transcription factors or transcriptional co-regulators such as FOXO, HNF-4 $\alpha$ and PGC-1 $\alpha$ that are also a target of SIRT1. Additionally, exercise and CR also positively regulate AMPK activity. All these measures decrease morbidity and mortality of SM.

It is important to study the life history of an individual to better understand pathologies since many adult metabolic and heart diseases, such as obesity, MS, type-2 diabetes, hypertension and arterial coronary diseases can be traced to early intrauterine development 


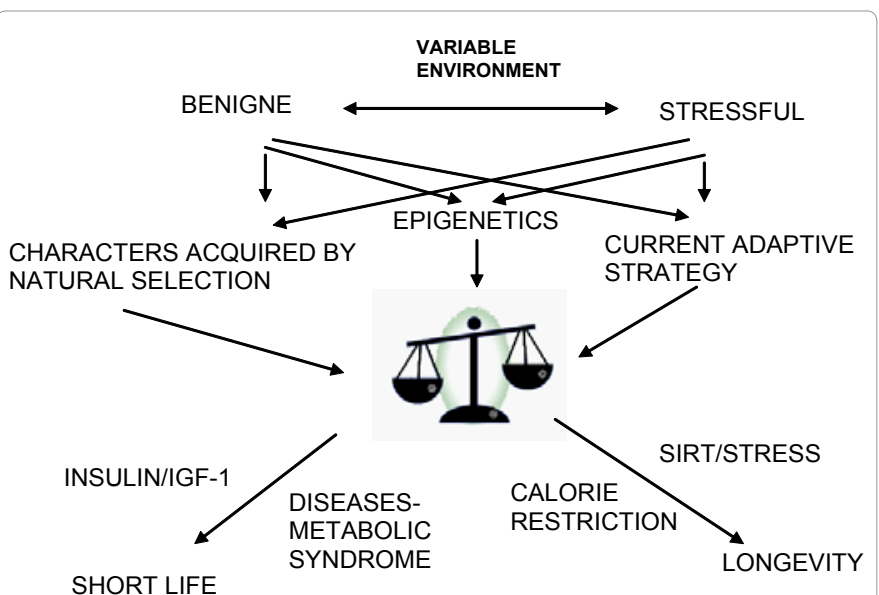

Figure 1: Variable environments determine acquired characteristics through natural selection, epigenetic expression and current adaptive strategies. These lead to activity in metabolic pathways which influence the rate of aging. Diseases such as metabolic syndrome also modulate the activity in these pathways and modulate longevity.

and childhood. The study of the diseases in aging individuals is also important since aging not only modifies their characteristics but it may accelerate the aging process. The aging process may also predispose to appearance of diseases. The aging of the world's population contributes to the growing prevalence of the MS.

\section{References}

1. Godfrey KM, Lillycrop KA, Burdge GC, Gluckman PD, Hanson MA (2007) Epigenetic mechanisms and the mismatch concept of the developmental origins of health and disease. Pediatr Res 61: 5R-10R.

2. Nathanielsz PW (2006) Animal models that elucidate basic principles of the developmental origins of adult diseases. ILAR J 47: 73-82.

3. Robertson KD (2005) DNA methylation and human disease. Nat Rev Genet 6: 597-610.

4. Kuroda A, Rauch TA, Todorov I, Ku HT, Al-Abdullah IH, et al. (2009) Insulin gene expression is regulated by DNA methylation. PLoS One 4: 6953

5. Kaikkonen MU, Lam MT, Glass CK (2011) Non-coding RNAs as regulators of gene expression and epigenetics. Cardiovasc Res 90: 430-440.

6. Tang X, Muniappan L, Tang G, Ozcan S (2009) Identification of glucoseregulated miRNAs from pancreatic $\{$ beta\} cells reveals a role for miR-30d in insulin transcription. RNA 15: 287-293.

7. Ho DH, Burggren WW (2010) Epigenetics and transgenerational transfer: a physiological perspective. J Exp Biol 213: 3-16.

8. Gluckman PD, Hanson MA, Buklijas T, Low FM, Beedle AS (2009) Epigenetic mechanisms that underpin metabolic and cardiovascular diseases. Nat Rev Endocrinol 5: 401-408.

9. Waterland RA, Michels KB (2007) Epigenetic epidemiology of the developmenta origins hypothesis. Annu Rev Nutr 27: 363-388.

10. Burdge GC, Hanson MA, Slater-Jefferies JL, Lillycrop KA (2007) Epigenetic regulation of transcription: a mechanism for inducing variations in phenotype (fetal programming) by differences in nutrition during early life? $\mathrm{Br} \mathrm{J}$ Nutr 97 1036-1046.

11. Aguayo-Mazzucato C, Sanchez-Soto C, Godinez-Puig V, Gutierrez-Ospina G Hiriart M (2006) Restructuring of pancreatic islets and insulin secretion in a postnatal critical window. PLoS One 1: 35.

12. Srinivasan M, Laychock SG, Hill DJ, Patel MS (2003) Neonatal nutrition: metabolic programming of pancreatic islets and obesity. Exp Biol Med (Maywood) 228: 15-23

13. Ozanne SE, Hales CN (2002) Early programming of glucosa-insulin metabolism Trends Endocrinol Metab13: 368-373.
14. Gluckman PD, Hanson MA, Beedle AS (2007) Non-genomic transgenerational inheritance of disease risk. Bioessays 29: 145-154

15. Du M, Yan X, Tong JF, Zhao J, Zhu MJ (2010) Maternal obesity, inflammation and fetal skeletal muscle development. Biol Reprod 82: 4-12.

16. Bode KA, Dalpke AH (2011) HDAC inhibitors block innate immunity Blood 117: 1102-1103.

17. Matouk CC, Marsden PA (2008) Epigenetic regulation of vascular endothelia gene expression. Circ Res 102: 873-887.

18. Cardinale JP, Sriramula S, Pariaut R, Guggilam A, Mariappan N, et al. (2010) HDAC inhibition attenuates inflammatory, hypertrophic, and hypertensive responses in spontaneously hypertensive rats. Hypertension 56: 437- 444

19. Neel JV (1962) Diabetes mellitus: a thrifty genotype rendered detrimental by "progress"? Am J Hum Genet 14: 353-362.

20. Hales CN, Barker DJ, Clark PM, Cox LJ, Fall C, et al. (1991) Fetal and infant growth and impaired glucose tolerance at age 64. BMJ 303: 1019-1022.

21. Amen-Ra N (2006) Humans are evolutionarily adapted to caloric restriction resulting from ecologically dictated dietary deprivation imposed during the PlioPleistocene period. Med Hypotheses 66: 978-984

22. Liberman LS (2003) Dietary, evolutionary, and modernizing influences on the prevalence of type 2 diabetes. Ann Rev Nutr 23: 345-377.

23. Fu Q, McKnight RA, Yu X, Callaway CW, Lane RH (2006) Growth retardation alters the epigenetic characteristics of hepatic dual specificity phosphatase 5 . FASEB J 20: E1441-E1450 2127-2129.

24. Joss-Moore LA, Metcalfe DB, Albertine KH, McKnight RA, Lane RH (2010) Epigenetics and fetal adaptation to perinatal events: Diversity through fidelity. J Anim Sci 88: E216-E222.

25. Fu Q, Yu X, Callaway CW, Lane RH, McKnight RA (2009) Epigenetics: intrauterine growth retardation (IUGR) modifies the histone code along the rat hepatic IGF-1 gene. FASEB J 23: 2438-2449.

26. Forsdahl A (1977) Are poor living conditions in childhood and adolescence an important risk factor for arteriosclerotic heart disease? Br J Prev Soc Med 31 91-95

27. Wadsworth ME, Cripps HA, Midwinter RE, Colley JR (1985) Blood pressure in a cohort at the age of 36 related to social and familial factors, smoking and body mass. Br Med J (Clin Res Ed) 291: 1534-1538.

28. Barker DJ, Osmond C, Golding J, Kuh D, Wadsworth ME (1989) Growth in utero, blood pressure in childhood and adult life, and mortality from cardiovascular disease. BMJ 298: 564-567.

29. Yajnik CS (2002) The lifecycle effects of nutrition and body size on adult adiposity, diabetes and cardiovascular disease. Obes Rev 3: 217-224.

30. Yajnik CS (2004) Early life origins of insulin resistance and type 2 diabetes in India and other asian countries. J Nutr 134: 205-210.

31. Schulz LO, Bennett PH, Ravussin E, Kidd JR, Kidd KK, et al. (2006) Effects of traditional and western environments on prevalence of type 2 diabetes in Pima Indians in Mexico and the U.S. Diabetes Care 29: 1866-1871.

32. Dabelea D, Pettitt DJ, Hanson RL, Imperatore G, Bennett PH, et. al. (1999) Brith weight, type 2 diabetes, and insulin resistance in Pima Indian children and young adults. Diabetes Care 22: 944-950

33. Ma L, Hanson RL, Que LN, Cali AM, Fu M, et al. (2007) Variants in ARHGEF11, a candidate gene for the linkage to type 2 diabetes on chromosome lq, are nominally associated with insulin resistance and type 2 diabetes in Pima Indians. Diabetes 56: 1454-1459

34. Mu JJ, Liu ZQ, Yang J, Ren J, Liu WM, et al. (2008) Association between higher blood pressure level in children and adult blood pressure: 17 years follow-up results. Zhonghua Xin Xue Guan Bing Za Zhi 36:229-231.

35. Li L, Law C, Power C (2007) Body mass index throughout the life-course and blood pressure in mid-adult life: a birth cohort study. J Hypertens 25: 12151223.

36. Raitakari OT, Juonala M, Viikari JS (2005) Obesity in childhood and vascular changes in adulthood: insights into the cardiovascular risk in young Finns Study. Int J Obes (Lond) 29: S101-S104.

37. Hardy R, Wadsworth ME, Langenberg C, Kuh D (2004) Birthweight, childhood growth, and blood pressure at 43 years in a British birth cohort. Int J Epidemio 33: $121-129$. 
38. Law CM, Shiell AW, Newsome CA, Syddall HE, Shinebourne EA, et al. (2002) Fetal, infant, and childhood growth and adult blood pressure: a longitudinal study from birth to 22 years of age. Circulation 105: 1088-1092.

39. Osmond C, Barker DJ (2000) Fetal, infant, and childhood growth are predictors of coronary heart disease, diabetes, and hypertension in adult men and women. Environ Health Perspect 108: 545-553

40. Berenson GS, Srinivasan SR, Hunter SM, Nicklas TA, Freedman DS, et al. (1989) Risk factors in early life as predictors of adult heart disease: the Bogalusa Heart Study. Am J Med Sci 298: 141-151.

41. Lucas A (1998) Programming by early nutrition. An experimental approach. $J$ Nutr 128: 401S-406S

42. Wells JC (2003) The thrifty phenotype hypothesis: thrifty offspring or thrifty mother? J Theor Biol 221: 143-161.

43. Waterland RA, Michels KB (2007) Epigenetic epidemiology of the developmental origins hypothesis. Annu Rev Nutr 27: 363-388.

44. Vaquero A, Reinberg D (2009) Calorie restriction and the exercise of chromatin Genes Dev 23: 1849-1869.

45. de Kreutzenberg SV, Ceolotto G, Papparella I, Bortoluzzi A, Semplicini A et al. (2010) Downregulation of the longevity-associated protein Sirtuin 1 in insulin resistance and metabolic syndrome: potential biochemical mechanisms. Diabetes 59: 1006-1015.

46. Fowden AL, Hill DJ (2004) Intra-uterine programming of the endocrine pancreas. Br Med J 60: 123-142.

47. Ohneda M, Johnson JH, Inman LR, Unger RH (1993) GLUT 2 function in glucose unresponsive beta cells of dexamethasone-induced diabetes in rats. J Clin 92: 1950-1956.

48. Blazquez E, Sugase M, Blazquez M, Foa PP (1974) Neonatal changes in the concentration of liver cAMP and of serum glucose, FFA, pancreatic insulin and glucagon in man and in the rat. J Lab Clin Med 83: 957-967.

49. Girard J (1990) Metabolic adaptations to change of nutrition at birth. Bio Neonate 58: 3-15.

50. Aalinkeel R, Srinivasan M, Kalhan SC, Laychock SG, Patel MS (1999) A dietary intervention (high carbohydrate) during the neonatal period causes islet dysfunction in rats. Am J Physiol Endocr Metab 277: E1061-E1069.

51. Aalinkeel R, Srinivasan M, Song F, Patel MS (2001) Programming into adulthood of islet adaptations induced by early nutritional intervention in the rat. Am J Physiol Endocrinol Metab 281: E640-E648.

52. Srinivasan M, Aalinkeel R, Song F, Lee B, Laychock SG, et al. (2000) Adaptive changes in insulin secretion by islets from neonatal rats raised on a high carbohydrate formula. Am J Physiol Endocrinol Metab 279: E1347-E1357.

53. Petrik J, Srinivasan M, Aalinkeel R, Cookell S, Arany E, et al. (2001) A long term carbohydrate diet causes an altered ontogeny of pancreatic islets of Langerhans in the neonatal rat. Pediatr Res 49: 84-92.

54. Breant B, Gesina E, Blondeau B (2006) Nutrition, glucocorticoids and pancreas development. Horm Res 65: 98-104.

55. Hill DJ, Duvillie B (2000) Pancreatic development and adult diabetes. Pediatr Res 48: 269-274.

56. Sandovici I, Smith NH, Nitert MD, Ackers-Johnson M, Uribe-Lewis S, et al (2011) Maternal diet and aging alter the epigenetic control of a promoterenhancer interaction at the Hnf4a gene in rat pancreatic islets. Proc Natl Acad Sci U S A 108: 5449-5454.

57. Nava P, Collados MT, Massó F, Guarner V (1997) Endothelin mediation of insulin and glucose induced changes in vascular contractility. Hypertension 30: 825-829.

58. Nava P, Guarner V, Posadas R, Perez I, Baños G (1999) Insulin-induced endothelin release and vasoactivity in hypertriglyceridemic and hypertensive rats. Am J Physiol 277 (Heart Circ Physiol 46): H399-H404.

59. Ikeda N, Nakajima R, Tsunoda T, Nakamura M, Sugi K (2007) Insulin resistance and acute coronary syndrome in the young Japanese population have a strong association. Heart Vessels 22: 165-169.

60. Fujii N, Tsuchihashi K, Sasao H, Eguchi M, Miurakami H (2008) Insulin resistance functionally limits endothelium-dependent coronary vasodilation in nondiabetic patients. Heart Vessels 23: 9-15.

61. Baños G, Carvajal K, Cardoso G, Zamora J, Franco M (1997) Vascular reactivity and effect of serum in a rat model of hypertriglyceridemia and hypertension. Am J Hypertens 10: 379-388.

62. Rubio-Ruiz ME, Vargas-Gonzalez A, Monter-Garrido M, Díaz-Díaz E, GuarnerLans V (2010) Aortic vaso-ractivity during a postnatal critical window of the pancreas in rats. Heart Vessels 25: 248-253.

63. Berenson GS, Srinivasan SR, Hunter SM, Nicklas TA, Freedman DS, et al. (1989) Risk factors in early life as predictors of adult heart disease: the Bogalusa Heart Study. Am J Med Sci 298: 141-51.

64. Grobbee DE, van Hooft IM, de Man SA (1990) Determinants of blood pressure in the first decades of life. J Cardiovasc Pharmacol 16: S71-74.

65. Watkins AJ, Lucas ES, Torrens C, Cleal JK, Green L, et al. (2010) Maternal low-protein diet during mouse pre-implantation development induces vascular dysfunction and altered renin-angiotensin-system homeostasis in the offspring Br J Nutr 103: 1762-1770.

66. Raitakari OT, Juonala M, Kähönen M, Taittonen L, Laitinen T, et al. (2003) Cardiovascular risk factors in childhood and carotid artery intima-media thickness in adulthood: the Cardiovascular Risk in Young Finns Study. JAMA 290: $2277-2283$

67. Bogdarina I, Welham S, King PJ, Burns SP, Clark AJL (2007) Epigenetic modification of the renin-angiotensin system in the fetal programming of hypertension. Circ Res 100: 520-526.

68. Porter JP, King SH, Honeycutt AD (2007) Prenatal high-salt diet in the SpragueDawley rat programs blood pressure and heart rate hyperresponsiveness to stress in adult female offspring. Am J Physiol Regul Integr Comp Physiol 293 R334-R342.

69. Kenyon CJ (2010) The genetics of aging. Nature 464: 504-512.

70. Fraga Mf, Esteller M (2005) Towards the human cancer epigenome: a first draft of histone modifications. Cell Cycle 4: 1377-1381.

71. Foley DL, Craig JM, Morley R, Olsson CJ, Dwyer T, et al. (2009) Prospects for epigenetic epidemiology. Am J Epidemiol 169: 389-400.

72. Schwer B, Schumacherb B, Lombarda DB, Xiaoc C, Kurtevd MV, et al. (2010) Neural sirtuin 6 (Sirt6) ablation attenuates somatic growth and causes obesity. Proc Natl Acad Sci U S A 107: 21790-21794.

73. Rincon M, Rudin E, Barzilai N (2005) The insulin/IGF-1 signaling in mammals and its relevance to human longevity. Exp Gerontol 40: 873-877.

74. Rozing MP, Westendorp RGJ, Frölich M, de Craen AJM, Beekman M, et al. (2009) Human insulin/IGF-1 and familial longevity at middle age. Aging (Albany NY) 1: 714-722.

75. Guarner V, Carbó R, Rubio ME, Baños de MacCarthy G (2005) Aging of the cardiovascular system. Benhagen EF. Nova Biomedical Books, USA.

76. Baños G, El Hafidi M, Pérez-Torres I, Guarner V (2009) Insulin resistance and the metabolic syndrome. Yao EB. Nova Biomedical Books. USA.

77. Guarner-Lans V, Rubio-Ruiz ME, Perez-Torres I, Baños de McCarthy G (2011) Relation of aging and sex hormones to metabolic syndrome and cardiovascula disease. Exp Gerontol 46: 517-523.

78. Fadini GP, Ceolotto G, Pagnin E, de Kreutzenberg S, Avogaro A (2011) At the crossroads of longevity and metabolism: the metabolic syndrome and lifespan determinant pathways. Aging Cell 10: 10-17.

79. Catalano KJ, Bergman RN, Ader M (2005) Increased susceptibility to insulin resistance associated with abdominal obesity in aging rats. Obes Res 13: 11 20.

80. Crosford R (1999) Insulin resistance, obesity and diabetes: the connection. $J$ Austr College Nutrit Environ Med 18: 3-10.

81. Dominguez LJ, Barbagallo M (2007) The cardiometabolic syndrome and sarcopenic obesity in older persons. J Cardiometab Syndr 2: 183-189.

82. Unger RH (2003) Lipid overload and overflow: metabolic trauma and the metabolic syndrome. Trends Endocrinol Metab 14: 398-403.

83. Qiang W, Weiqiang K, Qing Z, Pengju Z, Yi L (2007) Aging impairs insulinstimulated glucose uptake in rat skeletal muscle via suppressing AMPK alpha. Exp Mol Med 39: 535-43.

84. Wu D, Ren Z, Pae M, Guo W, Cui X, et al. (2007) Aging up-regulates expression of inflammatory mediators in mouse adipose tissue. J Immunol 179: 4829-4839. 
Citation: Veronica G, Esther RRM (2011) Metabolic Syndrome: Early Development and Aging. J Diabetes Metab S2:002. doi:10.4172/2155-6156. S2-002

85. Greenfield JR, Campbell LV (2006) Relationship between inflammation, insulin resistance and type 2 diabetes: 'cause or effect'? Curr Diabetes Rev 2: 195211.

86. De Martinis M, Franceschi C, Monti D, Ginaldi L (2005) Inflamm-ageing and lifelong antigenic load as major determinants of ageing rate and longevity. FEBS Lett 579: 2035-2039.

87. Grimble RF (2003) Inflammatory response in the elderly. Curr Opin Clin Nutr Metab Care 6: 21-29.

88. Bauer ME (2005) Stress, glucocorticoids and ageing of the immune system Stress 8: 69-83.

89. Pralong FP, Gaillard RC (2001) Neuroendocrine effects of leptin. Pituitary 4: 25-32.

90. Peters A, Schweiger U, Fruhwald-Schultes B, Born J, Fehm HL (2002) The neuroendocrine control of glucose allocation. Exp Clin Endocrinol Diabetes 110: 199-211.

91. Bjorntorp P, Rosmond R (2000) Neuroendocrine abnormalities in viscera obesity. Int J Obes Relat Metab Disord 24: S80-S85.

92. Bjorntorp P, Rosmond R (2000) The metabolic syndrome-a neuroendocrine disorder? Br J Nutr 83: S49-S57.

93. Stein-Behrens BA, Sapolsky RM (1992) Stress, glucocorticoids, and aging Aging (Milano) 4: 197-210.

94. Butcher SK, Lord JM (2004) Stress responses and innate immunity: aging as a contributory factor. Aging Cell 3: 151-160.

95. Kaaja RJ, Pöyhönen-Alho MK (2006) Insulin resistance and sympathetic overactivity in women. J Hypertens 24: 131-141.
96. Fliers E, Kreier F, Voshol PJ, Havekes LM, Sauerwein HP et al (2003) White adipose tissue: getting nervous. J Neuroendocrinol 15: 1005-1010.

97. Krier F, Fliers E, Voshol PJ, Van Eden CG, Havekes LM, et al. (2002) Selective parasympathetic innervation of subcutaneous and intra-abdominal fatfunctional implications. J Clin Invest 110: 1243-1250.

98. Froy O (2010) Metabolism and circadian rhythms-implications for obesity Endocr Rev 31: 1-24.

99. Nakamura TJ, Nakamura W, Yamazaki S, Kudo T, Cutler T, et al. (2011) Agerelated decline in circadian output. J Neurosci 31: 10201-10225.

100. Bellet MM, Sassone-Corsi P (2010) Mammalian circadian clock and metabolism - the epigenetic link. J Cell Sci 123: 3837-3848.

101. Sowers RJ (2004) Insulin resistance and hypertension. Am J Physiol Hear Circ Physiol 286: H1597-H1602.

102. Michelson RM Hypertension in the elderly.

103. Henriksen EJ (2007) Improvement of insulin sensitivity by antagonism of the renin-angiotensin system. Am J Physiol Regul Integr Comp Physiol 293: 974 980.

104. Soltis EE, Newman PS (1992) Ontogeny of vascular smooth muscle responsiveness in the postweaning rat. Dev Pharmacol Ther 18: 44-54.

105. Lee DH, Lee JU, Kang DG, Paek YW, Chung DJ, et al. (2001) Increased vascular endothelin-1 gene expression with unaltered nitric oxide synthase levels in fructose-induced hypertensive rats. Metabolism 50: 74-78.

106. Rubio ME, Baños G, Diaz E, Guarner V (2006) Effect of age on insulin-induced endothelin release and vasoreactivity in hypertriglyceridemic and hypertensive rats. Exp Geront 41: 282-288. 